\title{
INTERESES TRANSALPINOS DE JAIME II EN LA ÉPOCA DE LA CONQUISTA DEL REINO DE MURCIA. LA DONACIÓN DE LOS CALATRAVOS AL INFANTE JUAN EN 1304
}

\author{
Pedro Carlos PICATOSTE NAVARRO
}

En el verano de 1296 Jaime II de Aragón ocupa y gobierna el antiguo reino hudita de Murcia, lo que le hace entrar en conflicto con la otra gran fuerza cristiana peninsular, Castilla. Esta guerra por la posesión de las tierras levantinas se mantuvo por espacio de ocho años, hasta que en 1304, con la intervención de Dionís de Portugal, las coronas litigantes firmaron en Torrellas una paz que suponía el reparto del territorio en litigio'. Conseguida la paz con el castellano y el afianzamiento de la frontera sur de la Corona de Aragón ${ }^{2}$, Jaime II acometió nuevas empresas en uno de sus más continuados propósitos: seguir manteniendo la soberanía catalano-aragonesa en tierras de Nápoles y Sicilia, siendo en este terreno el control comercial del Mediterráneo el ob-

1 Acerca de la conquista del reino de Murcia por Jaime II cfr. ESTAL GUTIÉRREZ, J. M. del, Conquista y anexión de las tierras de Alicante, Elche, Orihuela y Guardamar al Reino de Valencia por Jaime Il de Aragón (1296-1308), Alicante, 1982. Id., Corpus Documental del reino de Murcia bajo la soberania de Aragón (1296-1304/5). Colección de Documentos Medievales Alicantinos 1/1, Alicante, 1985. Id., El reino de Murcia bajo Aragón (1296-1305). Colección documental del medievo alicantino. Corpus Documental 1/2, Alicante, 1990. FERRER i MALLOL, M. ${ }^{\text {a }}$ T., «Notes sobre la conquesta del regne de Múrcia per Jaume II (1296-1304), en Homenatge a la memòria del Prof. Emilio Sáez. Aplec d'estudis dels seus deixebles y col.laboradors, Barcelona, 1989, pp. 27-44. Mientras que al respecto del acuerdo de Torrellas cf. TORRES FONTES, J., La delimitación del sudeste peninsular (tratados de Torrellas-Elche, 1304-1305), Murcia, 1951. ESTAL GUTIÉRREZ, J. M. del, El reino de Murcia bajo Aragón .... $/ 1,1$, pp. 89-103 y doc. 213.

2 GUINOT i RODRÍGUEZ, E., Els límits del regne. El procés de formació territorial del País Valencià medieval (1238-1500), Valencia, 1995. 
jetivo fundamental ${ }^{3}$. Ello, además, en un momento especialmente delicado en este sector, por cuanto tanto la liga urbana de Barcelona como los mercaderes de la Corona de Aragón y el propio rey Justo se enfrentaban a importantes contratiempos consecuencia del proteccionismo y de la subida de aranceles de los capetos franceses y de su homónimo de Mallorca, que gravaba la actividad comercial en los «antepuertos» de Barcelona y Valencia, vitales para el comercio ultramarino de la Corona de Aragón ${ }^{4}$. Desde el siglo XIII son los mercaderes catalanes los que controlan el comercio en esta zona del Mediterráneo, viéndose consolidada tal preeminencia con el poder y dominio político de la Corona de Aragón en ese momento. Para estos mercaderes el reino de Nápoles era un mercado importante, tanto por el consumo como por ser un punto comercial de primer orden por sus ferias y lugar estratégico en el centro del Mare Nostrum. Se vendía a Nápoles lana, cueros, azafrán, cera, sal y paños catalanes, valencianos y mallorquines ${ }^{5}$. Además, para reafirmar esta actividad comercial los monarcas otorgaron privilegios a los mercaderes catalanes, que forman en la zona numerosas colonias.

Sin duda, en este contexto se producen una serie de hechos que afianzan la presencia aragonesa en ultramar. Uno de estos aspectos es analizado en la presente comunicación. Se trata de la donación efectuada por el maestre de la Orden de Calatrava, García López, en favor del infante Juan, hijo de Jaime II de Aragón, por la que el infante recibe una serie de posesiones en Italia; ello seis meses antes de la firma del tratado de Torrellas, aunque sin relación con él. García López concedía a mediados de febrero de 1304 al infante Juan, quien ya incluso antes de nacer había sido destinado a la carrera religiosa, una serie de posesiones que la Orden tenía en Italia ${ }^{6}$. Nombraba como procurador para encargarse del asunto a fray Joan de Sempere, calatravo también, quien tenía como misión poner en posesión de tales bienes al representante del joven infante, que por razón de su minoría de edad fue nombrado por su padre el rey: se trataba de Enric de Quintavall. El apelativo de familiarem nostrum con el que Jaime II denomina al caballero Quintavall nos hace pensar que se tratase de una persona del círculo real que gozaba de toda la confianza del monarca.

García López de Padilla, según la crónica de Calatrava impresa en Toledo en $1571^{7}$, fue electo maestre en el año 1296 pero no en concordia, ya que otros caballeros de la Orden eligieron antes a Gutierre Pérez. Esta disputa dio lugar a que cada uno de ellos se apoderase de los más pueblos y castillos de la Orden que pudieron, durando la crisis algo menos de cuatro años. Llegó el acuerdo tras la mediación de Roma, mientras tanto las posesiones de la Orden estuvieron bajo la jurisdicción del maestre de Alcántara. La solución daba la razón a García López, corriendo de esto el año de 1301.

3 MIRÓN, B.L., Las reinas de Aragón, sus vidas y sus épocas, Valencia.

4 RIERA i MELIS, A., La Corona de Aragón y el Reino de Mallorca en el primer cuarto del siglo XIV, Madrid-Barcelona 1986.

5 SALRACH MARÉS, J. M. a La Corona de Aragón: plenitud y crisis, Historia 16, Madrid 1996.

6 Apéndice documental, doc. n. $¹$.

7 RADES Y ANDRADA, F. de, Chronica de la orden y Cavalleria de Calatrava, Toledo, 1571. 
Hay que tener en cuenta que en tiempos de este maestre se producen importantes acontecimientos para las órdenes militares en el ámbito europeo. Como sabemos la Iglesia termina condenando a los templarios y sus bienes son confiscados ${ }^{8}$. La orden del Temple tenía un carácter marcadamente militar, si bien lo que provocó su fin fue su inmensa riqueza y la amplitud de su sistema crediticio, que la hicieron demasiado poderosa a ojos de monarquías como la francesa que precipitó su caída. Felipe IV el Hermoso inició el proceso contra los templarios en 1307 y Clemente V, aliado del capeto, los terminó disolviendo cinco años después, pasando sus bienes en Francia tras el expolio regio a los hospitălarios; ${ }^{9}$ el poder real se afianza sobre la milicia religiosa con la aquiescencia del Papa. En otros reinos los bienes del Temple se reparten entre órdenes autóctonas o de nueva creación, como Montesa en Aragón o las de Calatrava, Santiago y Alcántara en Castilla; el espíritu de la cruzada y la oposición al Islam eran sus fundamentos ${ }^{10}$.

Una de las consecuencias directas de esta decisión, como hemos visto, es la creación en el reino de Valencia de la Orden de Montesa dependiente de la de Calatrava ${ }^{11 .}$ La crónica antes citada señala de forma explícita que el rey de Aragón participó activamente en la petición al Papa de que los bienes templarios de esta zona bajo su jurisdicción pasasen a depender de Calatrava por medio de una nueva Orden que vigilase esas tierras, todavía frontera de las posibles razzias musulmanas. Jaime II de Aragón junto con Dionís de Portugal se opusieron a la ambición de los hospitalarios sobre las posesiones del Temple. La Orden de Montesa aceptó la regla de Calatrava y del Císter, pero para no someterse directamente a los designios del maestre calatravo, castellano, pasó a depender del monasterio cisterciense de Santes Creus en Tarragona. La petición del rey Jaime la hizo, según la mentada crónica, "por devoción que tuvo a esta orden de Calatrava, y afición particular al Maestre don Garci Lopez". El Papa concedió la petición en el año 1317, fundándose al año siguiente el Convento de San Jorge de la villa de Montesa, siendo su primer maestre Guillen Erilli2.

A lo que parece, existieron siempre muy buenas relaciones entre el calatravo García López y la Corona de Aragón, de lo contrario no se explicaría el hecho de que fuese Alcañiz, dentro del reino de Aragón, la población elegida por el maestre para refugiarse cuando años después huyó de Castilla. Sabemos igualmente que en 1328 García López asistió a los fastos de la coronación de Alfonso IV acompañado incluso de «gran cavalleria» ${ }^{13}$, siendo ésta su presencia última en acontecimiento importante antes de

8 MARTÍNEZ díEZ, G., Los templarios en la Corona de Castilla, Burgos, 1993.

9 BORDONOVE, G., Les templiers. Histoire et tragedie, París, 1974.

10 RUNCIMAN, S., Historia de las cruzadas, Madrid, 1994.

11 RIGALT y NICOLAS, B., Diccionario histórico de las órdenes de Caballería, Barcelona, 1858. GUINOT RODRÍGUEZ, E., Feudalismo en expansión en el norte valenciano. Antecedentes y desarroIlo del señorío de la Orden de Montesa. Siglos XIII y XIV, Castellón, 1986.

12 RIGALT y NICOLAS, B., Diccionario histórico ... Quien por cierto acudió a los actos de coronación de Alfonso el Benigno en la Aljafería de Zaragoza, donde fue armada caballero por en infante Pedro, hermano del rey. ZURITA, J., Anales de la Corona de Aragón, edición preparada por A. CANELLAS LÓPEZ, Zaragoza, 1972, 3, VII, I, pp. 300-301.

13 SOLDEVILA, F., Les quatre grans cròniques, Barcelona, 1971 
renunciar al maestrazgo tras casi tres décadas al frente de la orden de Calatrava. No obstante tal renuncia, García López se quedó con lo que la Orden tenía en la Corona de Aragón y con el castillo y encomienda de Zorita (1329). Parece evidente que Jaime II disfrutaba de un aliado en el seno de una de las órdenes militares más importantes y potentes con gran fuerza en Castilla.

En este estado de cosas es posible que las donaciones acreditadas en los documentos que aportamos pudiesen haber favorecido de modo notable esa "afición particular» por García López en el ánimo de Jaime II.

Los territorios cedidos al Infante Juan, todavía niño, por García López y la orden de Calatrava fueron Santo Angel de la Ossaria, Castelluço, Pons, Albaneti, Troya, Fraguinyano, Sanctus Pantalomeus, Romania, Astol, Foggia, Sancta María de Posse, Brindissi, Salpione, Sancta Maria la Mutata, La Grotayla, Maiolano, Sanctus Nichola, y las «piscarias» de Tarento, Avorna, Alexia, Sancta Elia, Ayora de Matreria et Sanctus Dimine.

El embajador elegido por Jaime II para tomar posesión de estas donaciones fue, lógicamente, alguien que en esos momentos gozaba de toda su confianza, Enric de Quintavall, a quien además de entregarle sus "vices", licencia para nombrar a procuradores, subalternos o removerlos cuando lo considerase oportuno, así como para hacer y deshacer a voluntad ${ }^{14}$, le arropó de toda una labor diplomática encaminada a facilitar su trabajo en aquellas tierras. Los documentos que se envían así lo acreditan. Sin dejar detalle al azar se giran misivas entre el 24 y el 30 de abril de 1304 a todos aquellos, clérigos y laicos, reyes y nobles, que Jaime II consideró personas influyentes que facilitarían la tarea al legado Quintavall. Carlos de Nápoles ${ }^{15}$; Benedicto XI de Roma ${ }^{16}$, quien además había de darle su bendición; Marco, diácono cardenal de Santa María in Portico; Laudulfo, diácono cardenal de Sant Angelo; Francesco, diácono cardenal de Santa María in Cosmedin; a Napoleón, diácono cardenal de San Adrián; al maestro Pedro Hispano, cardenal de la Sabina; Francesco Napoleón, diácono cardenal de Santa Lucía in Sílice ${ }^{17}$; Ramón Berenguer, hijo del rey de Jerusalem y Sicilia; Felipe, príncipe de Tarento; Roberto, duque de Calabria; la reina María de Jerusalen, Sicilia y Hungría ${ }^{18}$; Bartolomé Siginulfo, "comite tolesino», camarero del rey de Sicilia; Bartolomé de Capua «logotero" y protonotario del rey de Sicilia; Sergio Siginulfo, mariscal del rey de Sicilia; Diego de Lavat, caballero del duque de Calabria ${ }^{19}$; García, prior de Santa Cristina y procurador real de la Curia Romana; Vidal de Vilanova Curia Romana ${ }^{20}$; Roberto, duque de Calabria ${ }^{21}$; Pedro, obispo y canciller del rey de Sicilia; Giovanni Pipino, maestro racional del rey de Sicilia; a Roger de Lauria, almirante del

14 ACA, C, reg. 235. f. 60 v. (1304, abril, 30).

15 ACA, C, reg. 235, f. 61r. (1304, abril, 24 y 26).

16 ACA, C, reg. 235, f. 61 r. (1304, abril, 30).

17 ACA, C, reg. 235, f. 61 v. (1304, abril, 30).

18 ACA, C, reg. f. 61 v. (1304, abril, 26).

19 ACA, C, reg. 235, f. 62r. (1304, abril, 26).

20 ACA, C, reg. 235, f. 62r. (1304, abril, 26).

21 ACA, C, reg. 235, f. 62r. (1304, abril, 26). 
rey de Aragón; a Ermengando, conde de Ariano y maestro justiciero del rey de Sicilia; Ramón de Balso, domino Avellini; Guillermo, electo de Salerno, obispo, canciller y consejero del duque de Calabria'22; Manfredo, marqués de Saluciarum; Pedro de Victo "alme urbis perfecto"; y Porçello de filiis Urssi ${ }^{23}$ son objeto de sus cartas. A todos ellos se comunicó la razón del viaje de Quintavall y a todos ellos se solicitó ayuda de todo tipo, consejo y favor para su legado, que en algún caso los había de visitar, en atención a la amistad que se profesaban. La calidad de los receptores de las misivas dice mucho del interés del rey de Aragón por el asunto en cuestión.

Jaime II cuidó, a su vez, el recorrido que su representante había de hacer; con este fin escribió a Beltrán Jordan de la Ylla, senescal del rey de Francia, o a su lugarteniente, para que supiesen que Quintavall pasaría por las tierras del rey francés a la cabeza de una comitiva aragonesa montado en un caballo de pelo negro de su propiedad. Idéntica misiva dirigía el rey de Aragón a Ivo Girardi, rector de Montpellier ${ }^{24}$; al rey de Mallorca; a Ricardo de Cabatesa, senescal del rey Carlos ${ }^{25}$; a Cristiano Spinola y a Françesco Scarsafica ${ }^{26}$.

Por último, Jaime ll ordenó, por medio de documento, a todos sus oficiales y súbditos que ayudasen en aquello para lo que fuesen requeridos a Enric de Quintavall mientras estuviese en tierras de la Corona de Aragón. Como vemos, la amplitud de esta actividad diplomática denota la importancia que el monarca concede a la toma de posesión de estas donaciones de la Orden de Calatrava para su hijo el infante Juan.

Huelga referir que Quintavall fue una persona de toda confianza de Jaime II, así lo denotan los adjetivos empleados en los documentos como "dilectum militem", "familiarem nostrum» «familiarem dicti domini regis Aragonum», etc. Lo que desde luego no era es un desconocido, aunque sea muy poco lo que sepamos sobre él. Le sabemos en calidad de escudero cercano a Jaime II durante la campaña murciana de 1296, de quien obtuvo, en pleno proceso conquistador, unas casas situadas en la ciudad de Murcia y una heredad en el término de dicha ciudad compuesta por viñas y otras posesiones que habían sido propiedad de Pedro Ibáñez a quien le fueron confiscadas por ser rebelde al rey de Aragón. Todo ello por los servicios prestados y a perpetuidad con todos los derechos ${ }^{27}$.

Por otra parte, encontramos a Enric de Quintavall en asuntos relacionados con la compra venta de la alquería de Benimeli, en el término de Denia, y de Segària ${ }^{28}$. Estas disposiciones reflejan como Enric de Quintavall compra a Bernat Desclapers dicha

22 ACA.1304, Abril, 26.Alagón.

23 ACA, C, reg. 235, f. $62 \mathrm{v}$.

24 ACA, C, reg. 235, f. $62 \mathrm{v}$.

25 ACA, C, reg. 235, f. $62 v$.

26 ACA, C, reg. 235, f. 63r.

27 ACA, C, Reg. 194, f. 247 r-v. (1296, agosto, 3).

28 OLMOS Y CANALDA, E., Inventario de los pergaminos del Archivo de la Catedral de Valencia, Valencia 1961. 
alquería el 12 de agosto de $1306^{29}$, siendo confirmada tal adquisición por el propio Jaime II el día 16 del mismo mes $^{30}$. Vuelve a aparecer en diciembre el caballero Quintavall vendiendo la alquería a Pere Ronda ${ }^{31}$, panicerus domine regine, quien la vende a su vez a Jaime II, que la vuelve a vender al propio Enric de Quintavall el día después. En este documento se especifica que se venden las casas, campos, hierbas, etc. de la alquería de Benimeli entre lo perteneciente a Segària, una montaña, la rambla de Riusech, la alquería de Açenech, el Rafol y el río de Arobulata-Sagra, camino de Laguart ${ }^{32}$. Tales transmisiones se completan en otro documento ${ }^{33}$. En marzo de 1307 Enric de Quintavall se concierta con Bienvenida, hija de Guillem Garcia sobre esta alquería de Benimeli $i^{34}$, aunque desconozcamos como pudo quedar la cosa, pues en 1325 Quintavall recibió como donación del entonces procurador valenciano Bernat de Sarrià el mero y mixto imperio de Benimeli. Volvemos a localizar a Enric de Quintavall en junio de 1328 participando en la negociación que llevó a la nueva redacción de la carta de población de Ayora ${ }^{35}$. La última noticia vinculada con este personaje tiene fecha de cinco años después, 1330, por la que queda encargado junto con Ramon Giner de hacer efectivo el pago determinado por Alfonso IV a los escribanos de la Procuración del reino de Valencia ${ }^{36}$.

29 Ibidem.

30 lbidem.

31 Ibidem.

32 lbidem.

33 lbidem.

34 lbídem.

35 Cf. FERRER i MALLOL, M." T., "La carta de població dels sarraïns de la Vall d'Aiora (1328)", en Sharq al-Andalus, 3, Alicante, 1986, pp. 89-94. GUINOT RODRÍGUEZ, E., Cartes de poblament medievals valencianes, Valencia, 1991, doc. $n .{ }^{\circ} 261$, pp. 495-501.

36 CABEZUELO PLIEGO, J. V., La Curia de la Procuración. Estructura de una magistratura medieval valenciana, en prensa. Gentileza del autor. 


\section{APÉNDICE DOCUMENTAL}

1304, febrero, 15. Valencie.

Donación de los calatravos al infante Juan de ciertas posesiones en Italia. ACA, C, reg. 235, f. 60r.

\section{Domini infantis Joannis ${ }^{37}$}

Carte et littere tradite Enrico de Quintavalle, missa ad partes Neapolis et Romane Curie pro habenda possessione bonorum que magistro Calatrave dedit inclito domino infanti Johanni, nato domini regis, que sunt in Apulia et Principatu et Romani ipsisque colligendis et ministrandis.

Noverint universi presentes litteras inspecturi quod nos cum, frater Garsias Lupi, magister Milicie de Calatrava, Ordinis Cisterciensis, ad devocionis affectum quem illustris et magnificus princeps dominus Jacobus, Dei gracia rex Aragonum, suique progenitores ad nostrum Ordinem habuerunt actenus, dederimus et concesserimus inclito domino infanti Johanni, prefati domini regis Aragonum nato, quandiu sibi fuerit vita comes omnia loca omnesque redittus, responsiones, fructus et universos proventus et iura quos et que ordo predictus de Calatrava recepit aut debet recipe seu recipere consuevit vel usus est recipere per Apuliam et Principatum ac Romanium in locis ipsis que infra scriptis nominibus nuncupantur et in terminis et terris et pertinenciis eorumdem que quidem loca hec sunt videlicet Sanctus Angelus de la Ossaria, Castelluço, Pons, Abbaneti, Troya, Fraguinyano, Sanctus Pantalameus, in Romania, Astol, Fogia, Sancta Maria de Posse, Brundusii, Salpione, Sancta Maria la Mutata, La Grotayla, Maiolano, Sanctus Nichola, eadem piscaria que est in Tarento, due piscaria altera in Avorna et altera in Alexna, Sancta Elia, Aoyra de Matreria et Sanctus Dimitre. Idcirco volentes huiusmodi concessionem nostram ad effectum produci, constituimus, facimus et ordinamus certum nuncium ac procuratorem nostrum vos, fratrem Johannem de Sancto Petro, priorem decimus Valencie, nostri Ordinis, ad inducendum loco nomine et pro parte nostra in possessionem corporalem predictorum omnium et singularium percipiendorum discretum Enrricum de Quintavalie, familiarem dicti domini regis Aragonum, quem idem dominus rex Aragonum nomine et pro parte dicti infantis, nunc pupilli, duxit ad hec cum carta sua specialiter deputandum. Ita siquidem quod ex tunc vasalli dicti Ordinis et alii quicumque astricti vel debentes de predictis vel aliquo predictorum nobis vel Ordini nostro modo aliquo respondere de ipsis omnibus et singulis dicto domino regi Aragonum vel cui voluerit donec dictus infans ad etatem pervenerit pubertatis et ex tunc ipsi infanti vel cui seu quibus voluerit integre respondeant et plenarie satisfaciant, sicut nobis Ordini tenebantur. Nos et enim, ut vasalli ipsi et alii, hec faciant, compleant et attendant per presentem cartam nostram eisdem mandatum facimus specialiter ratum insuper habemus et firmum et in futurum habere promittimus quicquid

37 Letra no contemporánea. 
per dictum fratrem Johannem, nuncium et procuratorem nostrum, circa hec acta fuerint sive gesta. In cuius attamen rei testimonium ad cautelam presentem cartam inde fieri fecimus sigilli nostri pendentis munimine roboratam.

Data Valencie, XVIำkalendas marcii, anno Domini $\mathrm{M}^{\circ} \mathrm{CCC}^{\circ}$ tercio.

Yo, Garçi la fis escrivir por mandado del maestre. 